\title{
Pulsed-light inactivation of pathogenic and spoilage bacteria on cheese surface
}

\author{
J. Proulx, ${ }^{*}$ L. C. Hsu, ${ }^{*}$ B. M. Miller, ${ }^{*}$ G. Sullivan, ${ }^{*}$ K. Paradis,† and C. I. Moraru*1 \\ *Department of Food Science, Cornell University, Ithaca, NY 14853 \\ †Department of Food Science and Agricultural Chemistry, McGill University, Ste Anne de Bellevue, Québec, H9X 3V9, Canada
}

\begin{abstract}
Cheese products are susceptible to postprocessing cross-contamination by bacterial surface contamination during slicing, handling, or packaging, which can lead to food safety issues and significant losses due to spoilage. This study examined the effectiveness of pulsed-light $(\mathrm{PL})$ treatment on the inactivation of the spoilage microorganism Pseudomonas fluorescens, the nonenterohemorrhagic Escherichia coli ATCC 25922 (nonpathogenic surrogate of Escherichia coli O157:H7), and Listeria innocua (nonpathogenic surrogate of Listeria monocytogenes) on cheese surface. The effects of inoculum level and cheese surface topography and the presence of clear polyethylene packaging were evaluated in a full factorial experimental design. The challenge microorganisms were grown to early stationary phase and subsequently diluted to reach initial inoculum levels of either 5 or $7 \log \mathrm{cfu} /$ slice. White Cheddar and process cheeses were cut into $2.5 \times 5 \mathrm{~cm}$ slices, which were spot-inoculated with $100 \mu \mathrm{L}$ of bacterial suspension. Inoculated cheese samples were exposed to PL doses of 1.02 to $12.29 \mathrm{~J} / \mathrm{cm}^{2}$. Recovered survivors were enumerated by standard plate counting or the most probable number technique, as appropriate. The PL treatments were performed in triplicate and data were analyzed using a general linear model. Listeria innocua was the least sensitive to PL treatment, with a maximum inactivation level of $3.37 \pm 0.2 \mathrm{log}$, followed by $P$. fluorescens, with a maximum inactivation of 3.74 \pm 0.8 log. Escherichia coli was the most sensitive to $\mathrm{PL}$, with a maximum reduction of $5.41 \pm 0.1 \mathrm{log}$. All PL inactivation curves were nonlinear, and inactivation reached a plateau after 3 pulses $\left(3.07 \mathrm{~J} / \mathrm{cm}^{2}\right)$. The PL treatments through UV-transparent packaging
\end{abstract}

Received February 1, 2015.

Accepted May 23, 2015.

${ }^{1}$ Corresponding author: cim24@cornell.edu and without packaging consistently resulted in similar inactivation levels. This study demonstrates that PL has strong potential for decontamination of the cheese surface.

Key words: pulsed light, surface decontamination, spoilage and pathogenic bacteria, Cheddar cheese, process cheese

\section{INTRODUCTION}

According to the US Centers for Disease Control (CDC), 90 food poisoning outbreaks related to cheese consumption were reported in the United States from 1998 to 2011, $49 \%$ of which were caused by cheese made from pasteurized milk (Gould et al., 2014). These outbreaks were primarily caused by postpasteurization cross-contamination of cheese when subjected to cutting, slicing, or packaging, either at the processing plant or in retail environments. These outbreaks involved a variety of cheeses, such as Hispanic-style cheeses (Jackson et al., 2011), Gorgonzola cheese (Koch et al., 2010), and ricotta salata (CDC, 2012). In the last example, surface cross-contamination tripled the extent of damage caused by the source, a single contaminated ricotta salata wheel: out of 22 people infected, only 7 were found to have directly consumed the cheese responsible for the outbreak. The recurring pathogens in these outbreaks were Listeria monocytogenes, a ubiquitous, psychrotolerant, gram-positive bacterium, and Escherichia coli O157:H7, a gram-negative bacterium commonly associated with fecal contamination.

Microbial contamination of cheese surface can also involve spoilage microorganisms, which cause undesirable changes in appearance, texture, flavor, and odor. Such deterioration of product quality leads to lower consumer acceptance, shorter shelf life, and significant losses for cheese makers. Pseudomonas spp., frequent spoilage bacteria in both raw and pasteurized milk (Ternström et al., 1993), are among the most common microorganisms implicated in food spoilage in general. Pseudomonas fluorescens has, for instance, caused a recall of 70,000 contaminated mozzarella balls in Italy 
(Nogarol et al., 2013) and a recall of Latin-style fresh cheese (queso fresco) exhibiting blue discoloration in the United States (Martin et al., 2011).

The main approach to avoiding postpasteurization microbial contamination of cheese is compliance with good manufacturing practices and proper sanitation. Nonetheless, given the high number of outbreaks involving pasteurized milk cheeses still occurring, an additional inactivation step that targets the contaminating microflora after processing, handling, and packaging could be extremely beneficial. Pulsed-light (PL) treatment, consisting of short, high-energy pulses of broad-spectrum light, could help address this problem. The high-intensity pulses are generated by a xenon lamp that converts the high-voltage, high-peak current to light in a wavelength range from 200 to $1,100 \mathrm{~nm}$ in milli- to microseconds (Dunn et al., 1995; Gomez-Lopez et al., 2007). The PL treatment has been approved by the US Food and Drug Administration as a process aid for the decontamination of food and food contact surfaces since 1996 (US FDA, 1996). It is a nondestructive treatment with very short treatment times, which makes it convenient to use in manufacturing, distribution, and retail environments. Pulsed light is also a nonthermal technology that leaves no chemical residue (Pereira and Vicente, 2010), which is compatible with current clean labeling trends.

The microbicidal effect of PL treatment is primarily attributed to the UV portion of its spectrum that causes formation of pyrimidine dimers within the bacterial DNA, which block DNA transcription and replication, ultimately leading to cell death (Wang et al., 2005; Elmnasser et al., 2007; Woodling and Moraru, 2007; Kramer and Muranyi, 2014). Several studies have shown that PL can effectively inactivate microorganisms on various foods and food contact surfaces, including 2-log reduction of $L$. innocua on fresh-cut mushrooms (Ramos-Villarroel et al., 2012), 2- to 4.5-log reduction of E. coli in apple juice (Sauer and Moraru, 2009; Palgan et al., 2011), 1-log reduction of Salmonella Typhimurium on beef and tuna carpaccio (Hierro et al., 2012), and >7-log reduction of L. innocua on low density polyethylene (Ringus and Moraru, 2013).

The objective of this study was to examine the effectiveness of PL for inactivating relevant spoilage and pathogenic microorganisms on cheese surface, including surrogates of L. monocytogenes and E. coli O157:H7 and the spoilage microorganism $P$. fluorescens. The effect of inoculum level, 2 different cheese surfaces, PL dose, and the presence of UV-transparent low-density polyethylene packaging on PL inactivation were evaluated in a full-factorial design.

\section{MATERIALS AND METHODS}

\section{Inoculum Preparation}

The challenge microorganisms for this study included L. innocua FSL C2-008 obtained from the Food Safety Laboratory at Cornell University (Ithaca, NY). This microorganism has been demonstrated to behave as a surrogate for L. monocytogenes in PL treatment (Uesugi and Moraru, 2009; Lasabagaster and Martinez de Marañon, 2012). The second challenge microorganism was the gram-negative E. coli ATCC 25922, a demonstrated surrogate for pathogenic E. coli O157:H7 for light-based treatments (Sauer and Moraru, 2009; Miller et al., 2012). The third organism was P. fluorescens 1150 , a gram-negative rod associated with milk spoilage with the ability to synthesize a fluorescent blue pigment, pyoverdin, and to form biofilms.

A single colony of each bacterial strain was isolated from tryptic soy agar plates and inoculated into $10 \mathrm{~mL}$ of tryptic soy broth for E. coli and P. fluorescens, or brain heart infusion for L. innocua. Cultures of $E$. coli and $L$. innocua were grown at $37^{\circ} \mathrm{C}$ with shaking at $225 \mathrm{rpm}$ until stationary phase, typically for 20 to 24 h, whereas $P$. fluorescens was grown at $30^{\circ} \mathrm{C}$ with shaking at $225 \mathrm{rpm}$ for 24 to $26 \mathrm{~h}$. The stationary phase cultures were then diluted in Butterfield's phosphate buffer to obtain initial inoculum levels of 5 or $7 \log$ cfu per cheese slice. These concentrations are much higher than realistic postprocessing contamination levels, but they were chosen to allow the generation of observable inactivation curves.

\section{Substrate Preparation}

Sharp white Cheddar cheese (Heluva Good, Sodus, NY) and white American singles (Kraft Foods Inc., Northfield, IL) purchased from a local retail store were chosen as cheese substrates because of their different surface characteristics. A sterilized cheese cutter was used to obtain $2.5 \times 5 \mathrm{~cm}$ (width $\times$ length) rectangular cheese slices of 1 to $3 \mathrm{~mm}$ thickness. The slices were individually spot-inoculated onto one face with the appropriate cell suspension using 10 droplets of $10 \mu \mathrm{L}$ each. The inoculated cheese samples were subsequently stored at $4^{\circ} \mathrm{C}$ in individual sterile Petri dishes overnight to allow the liquid portion of the inoculum to evaporate.

For the experiments performed through packaging, low-density polyethylene (LDPE; 2 mil polybags \#S951, Uline, Pleasant Prairie, WI) was used for Cheddar cheese; the original LDPE commercial plastic wrapper was used for process cheese. Packaging materials were 
cut to the same dimensions as the cheese samples, disinfected with $95 \%$ ethanol, air-dried, and then placed on top of the inoculated cheese slices immediately before PL treatment.

\section{PL Treatment}

The PL treatments were conducted at room temperature using a Xenon RS-3000C bench top unit (Xenon Corporation, Wilmington, MA) equipped with a xenon flash lamp emitting 360- $\mu$ s pulses at a frequency of 3 pulses/s. A single slice of cheese was placed centrally on an adjustable stainless steel shelf inside the PL unit at $5.8 \mathrm{~cm}$ from the surface of the lamp housing and subjected to $1,3,6,9$, or 12 pulses, corresponding to fluence levels of $1.02,3.07,6.14,9.22$, and $12.29 \mathrm{~J} /$ $\mathrm{cm}^{2}$, respectively. Inoculated cheese slices that were not exposed to PL were used as positive controls.

\section{Fluence Measurements}

To monitor the PL dose, fluence measurements were taken using a pyroelectric head (PE25BBH) coupled with a Nova II display (Ophir Optronics Inc., Wilmington, MA). During the measurement, the pyroelectric head and its chord were covered in aluminum foil, leaving only a $1-\mathrm{cm}^{2}$ circular opening exposed. Cumulative exposure was measured at a distance of $5.8 \mathrm{~cm}$ from the lamp housing, at a pulse width setting of $1.0 \mathrm{~ms}$. Measurements for 1, 3, 6, 9, and 12 pulses were taken in triplicate. At least 1 min was allowed between measurements to prevent overheating of the pyroelectric head.

\section{Recovery and Enumeration of Survivors}

The treated cheese slices, along with the packaging material for the treatments performed through packaging, were placed into sterile Whirlpak stomacher bags (Nasco, Fort Atkinson, WI) containing 1:10 (mass:volume) Butterfield's phosphate buffer solution, and stomached for $2 \mathrm{~min}$ at $230 \mathrm{rpm}$ using a Seward Stomacher 400 Circulator (Seward, Davie, FL). Appropriate dilutions of the stomached samples were plated in duplicate on selective media (BD Difco, Bedford, MA), as follows: MacConkey agar for E. coli, modified Oxford agar for L. innocua, and Pseudomonas isolation agar for $P$. fluorescens. A minimum incubation time of $24 \mathrm{~h}$ at $37^{\circ} \mathrm{C}$ was used for L. innocua and E. coli, and $24 \mathrm{~h}$ at $30^{\circ} \mathrm{C}$ for P. fluorescens. Preliminary tests demonstrated that an enrichment step was not necessary. Survivors were enumerated by SPC and survivor ratios were quantified as $\log \left(\mathrm{N} / \mathrm{N}_{0}\right)$, where $\mathrm{N}$ represents the number of recovered survivors (log cfu) and $\mathrm{N}_{0}$ repre- sents the measured initial inoculum (log cfu). When plate counts fell below $30 \mathrm{cfu} /$ plate, the 3 -tube most probable number technique, as described by Downes et al. (2001), was used. All experiments were performed in triplicate.

\section{Substrate Surface Characterization}

Water Contact Angle Measurements. Dynamic water contact angles were measured using a Ramé-Hart 500 Advanced Goniometer/Tensiometer (Ramé-Hart Inc., Succasunna, NJ) equipped with an automated dispensing system. The data were analyzed using the instrument's DROPimage software. Measurements were performed on $1 \mathrm{~cm} \times 1 \mathrm{~cm}$ cheese samples, using the sessile drop method. As a microsyringe filled with deionized ultra-pure water (Milli-Q, Merck Millipore Ltd., Billerica, MA) dispensed a $15-\mu \mathrm{L}$ droplet on the sample, 40 stable advancing left and right angles were calculated and averaged. All measurements were performed in triplicate and average values of left and right contact angles were used as a measure of surface hydrophobicity. Values smaller than $90^{\circ}$ indicate a hydrophilic surface, and values larger than $90^{\circ}$ indicate a hydrophobic surface. This measure of hydrophobicity was used to evaluate the potential for surface spreading of the liquid inoculum.

Surface Roughness Analysis. Surface roughness of the cheese substrates was determined to evaluate the potential for microbial hiding and light shading. Measurements were performed using a MicroXAM optical interferometric profiler with the MapVue mapping and analysis software (ADE Phase Shift Inc., Tucson, AZ), at the Cornell Center for Materials Research (Ithaca, NY). The following roughness parameters were determined: the average roughness $\left(\mathrm{S}_{\mathrm{a}}\right)$, the difference between the highest and lowest peak $\left(\mathrm{S}_{\mathrm{z}}\right)$, the surface skewness with values around zero indicating as many peaks as valleys $\left(S_{\mathrm{sk}}\right)$, and the surface kurtosis $\left(\mathrm{S}_{\mathrm{ku}}\right)$, with a value of 3 indicating a Gaussian peak height distribution. Measurements were conducted on $2.5 \times$ $3.4 \mathrm{~mm}$ sample surfaces, in triplicate, for each type of cheese.

\section{Statistical Analysis}

The data were analyzed with the general linear model up to a 3-way ANOVA using the statistical package JMP Pro 10 (SAS Institute Inc., Cary, NC). The model included microorganism type, inoculum level, cheese type, presence of packaging, and PL dose, with every full factorial interaction term as fixed effects, and replicates as a random variable. Backward variable selection 
a) $\quad-\circ-5 \log$, No packaging

a) $-5 \log$, With packaging

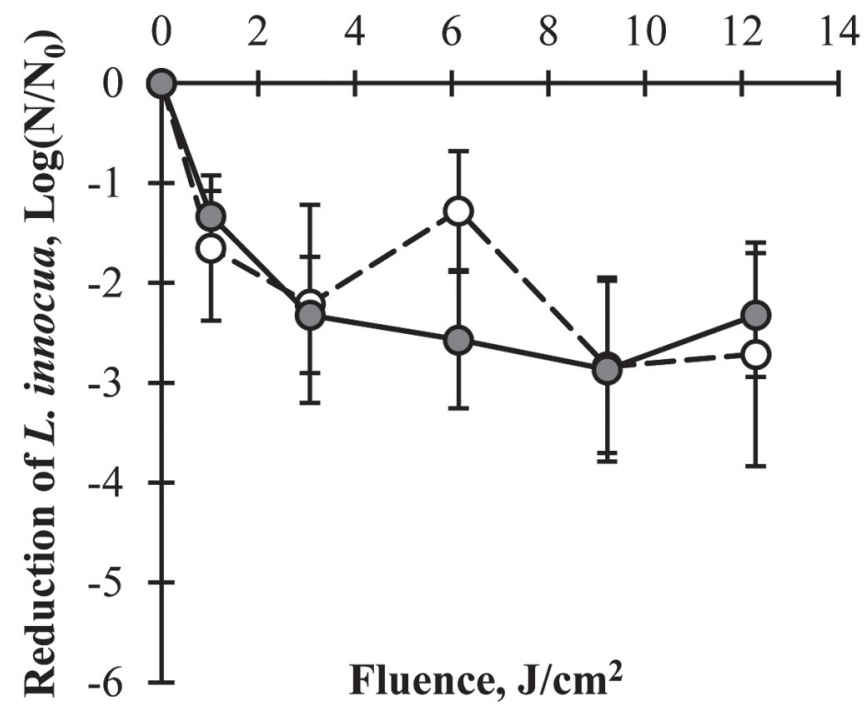

c)

$$
\begin{aligned}
& \text { - }-5 \log \text {, No packaging } \\
& -\begin{array}{r}
-5 \\
-
\end{array} \text { log, With packaging }
\end{aligned}
$$

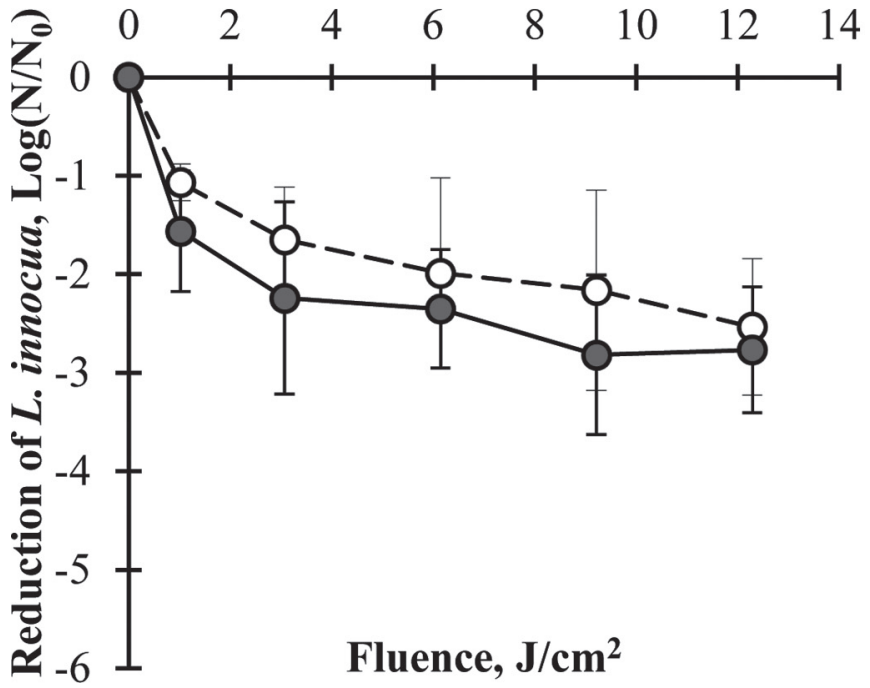

b)

$-\Delta-7 \log$, No packaging

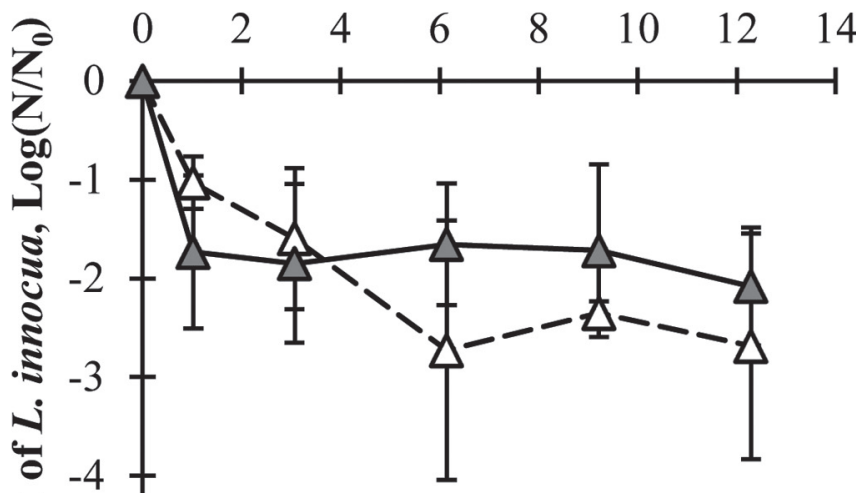

d)

$-\Delta-7 \log$, No packaging

$\triangle \triangle 7 \mathrm{log}$, With packaging

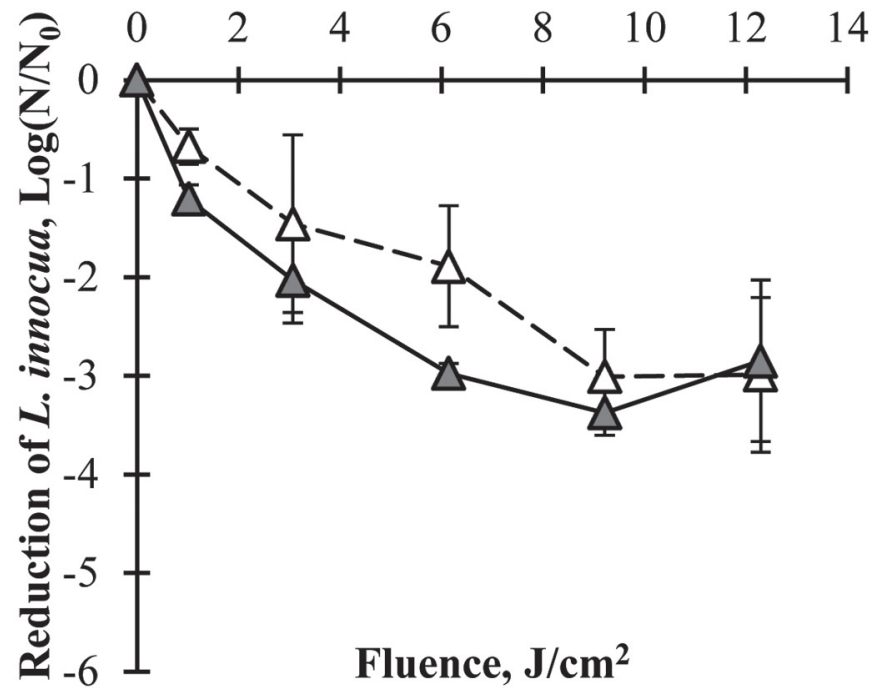

Figure 1. Pulsed-light inactivation of Listeria innocua on (a, b) Cheddar cheese and (c, d) process cheese, highlighting different combinations of initial inoculum levels ( $5 \mathrm{log}$ or $7 \mathrm{log} \mathrm{cfu} /$ slice) and presence (filled symbols, continuous line) or absence (open symbols, dotted line) of UV-transparent low-density polyethylene packaging. Survivor ratios were quantified as $\log \left(\mathrm{N} / \mathrm{N}_{0}\right)$, where N represents the number of recovered survivors $(\log \mathrm{cfu})$ and $\mathrm{N}_{0}$ represents the measured initial inoculum (log cfu). The data points represent mean values of triplicates and the error bars \pm 1 standard deviation.

was performed and Tukey's Honest Significant Difference (HSD) test was carried out on every significant effect to gain further insights on which factor level was most influential. The data for each microorganism were also analyzed separately.

\section{RESULTS AND DISCUSSION}

All the PL inactivation curves in this study exhibited similar characteristics; namely, a nonlinear inactivation curve and a plateau reached after 3 pulses $\left(3.07 \mathrm{~J} / \mathrm{cm}^{2}\right.$; 


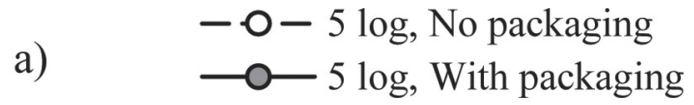

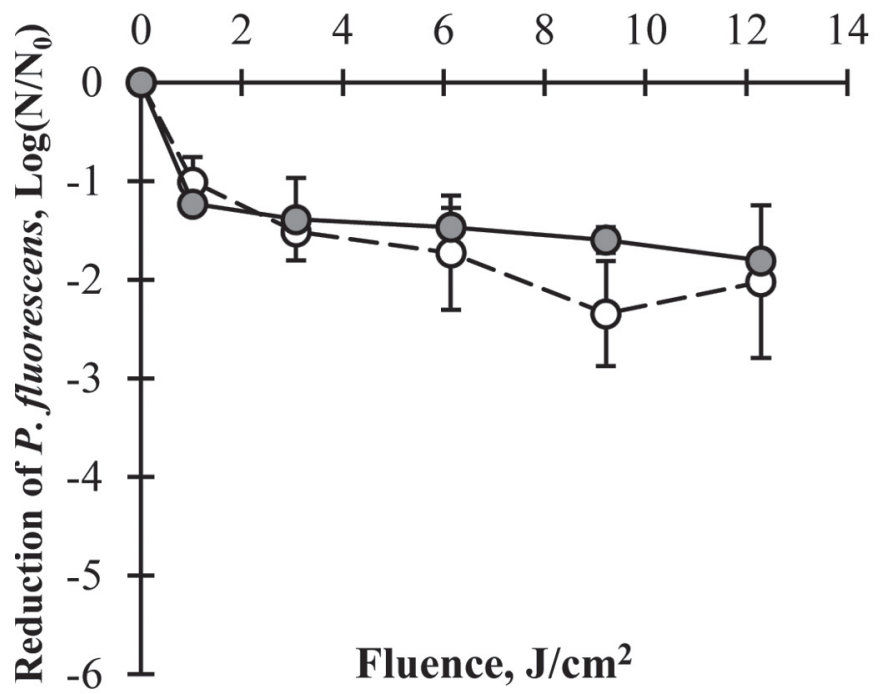

c) - $0-5 \log$, No packaging - 5 log, With packaging

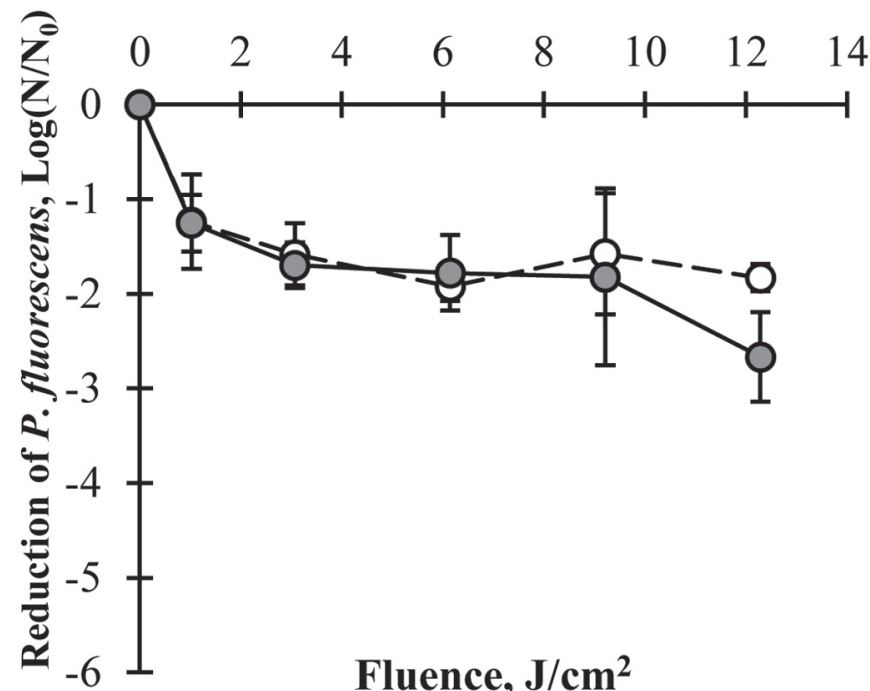

b) $-\Delta-7 \log$, No packaging $\triangle \longleftarrow 7 \log$, With packaging

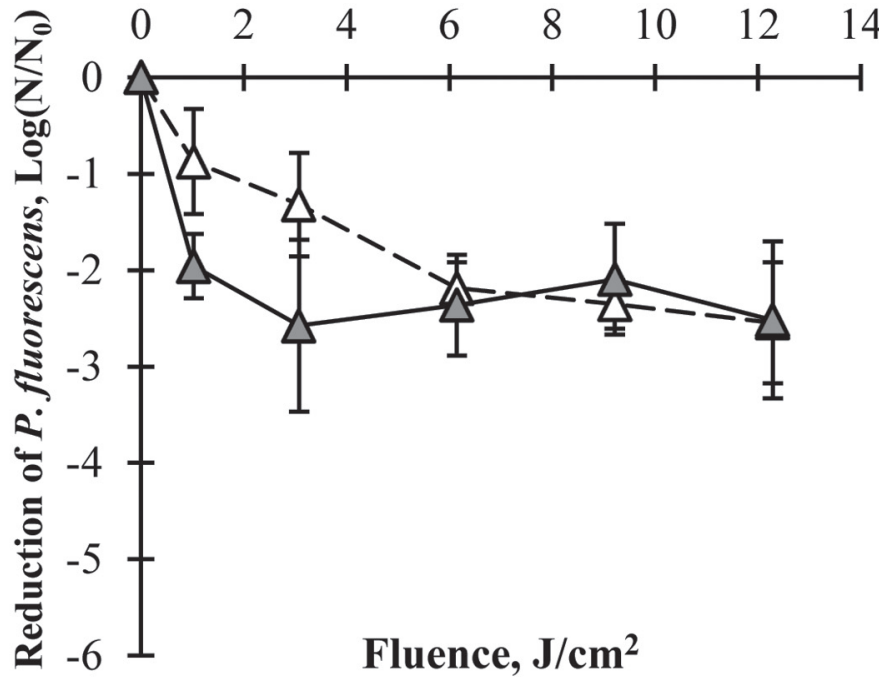

$-\Delta-7 \log$, No packaging

d)

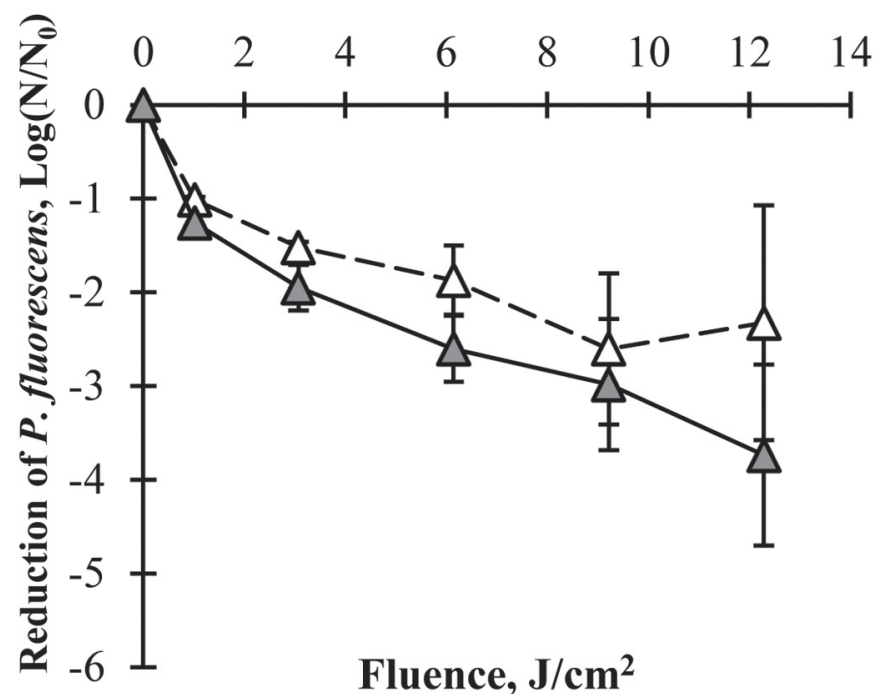

Figure 2. Pulsed-light inactivation of Pseudomonas fluorescens on (a, b) Cheddar cheese and (c, d) process cheese, highlighting different combinations of initial inoculum levels ( $5 \mathrm{log}$ or $7 \mathrm{log} \mathrm{cfu} /$ slice) and presence (filled symbols) or absence (open symbols) of UV-transparent lowdensity polyethylene packaging. Survivor ratios were quantified as $\log \left(\mathrm{N} / \mathrm{N}_{0}\right)$, where $\mathrm{N}$ represents the number of recovered survivors (log cfu) and $\mathrm{N}_{0}$ represents the measured initial inoculum (log cfu). The data points represent mean values of triplicates and the error bars indicate \pm 1 standard deviation..

Figures 1, 2, and 3). Levels of inactivation of at least 3-log reduction were achieved for all microorganisms and treatment conditions tested, at doses below $12 \mathrm{~J} /$ $\mathrm{cm}^{2}$, which is the maximum PL dose allowed by the Food and Drug Administration (US FDA, 1996).

\section{PL Inactivation on Cheese Surface: Effect of Microorganism Type}

Statistical analysis showed significantly different responses to PL by each of the microorganisms tested $(P$ 

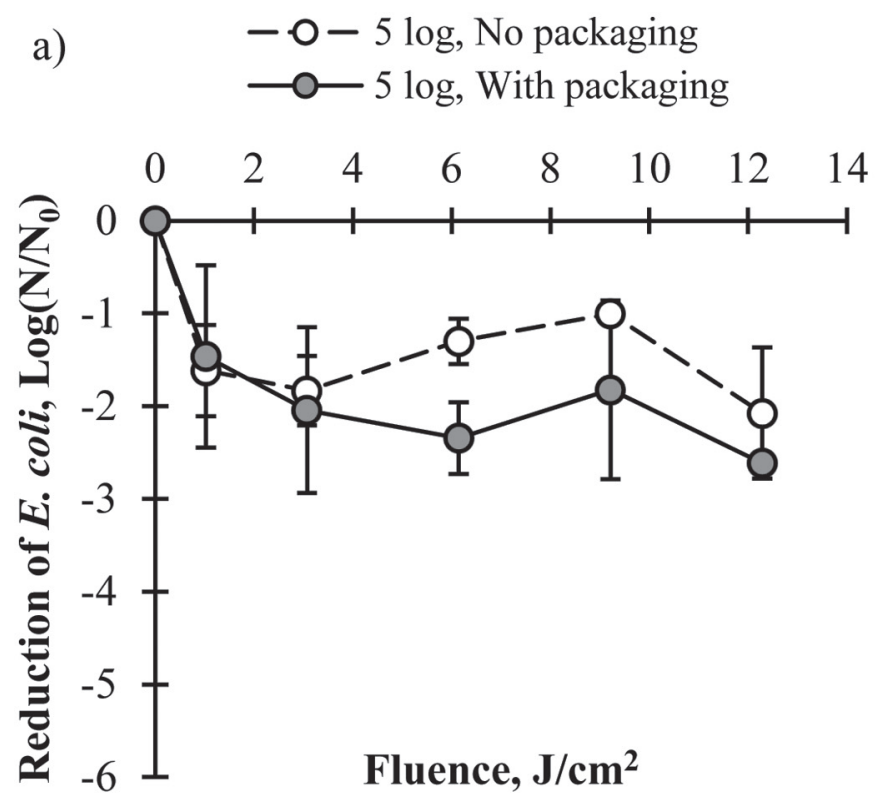

c)

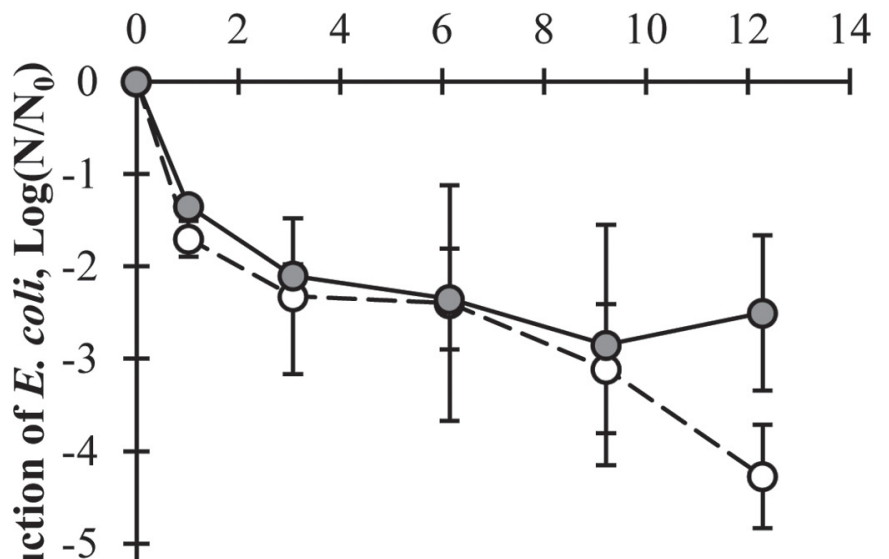

b) $-\Delta-7 \log$, No packaging
$-\Delta-7 \log$, With packaging

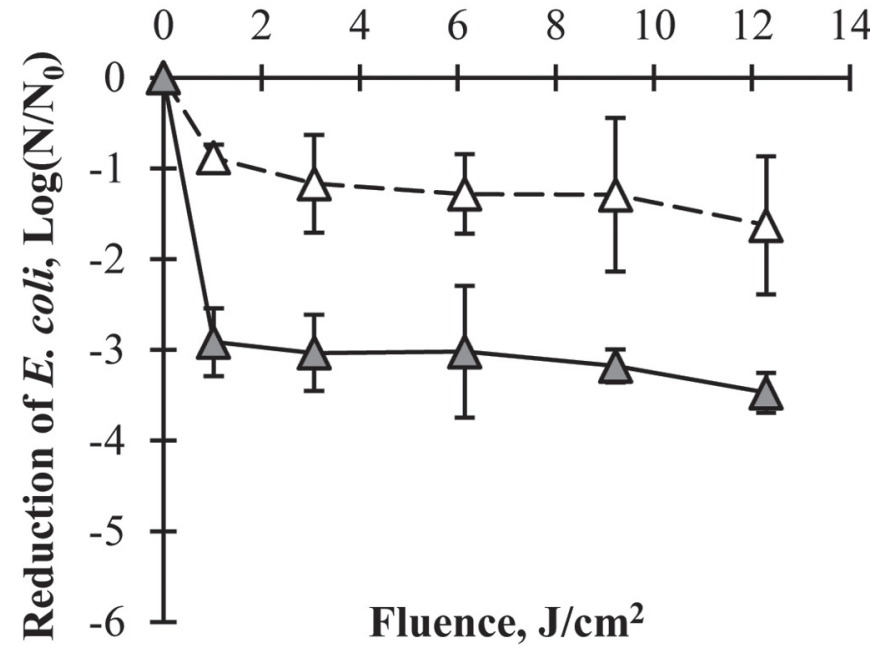

d) $-\Delta-7 \log$, No packaging $\triangle-7 \mathrm{log}$, With packaging

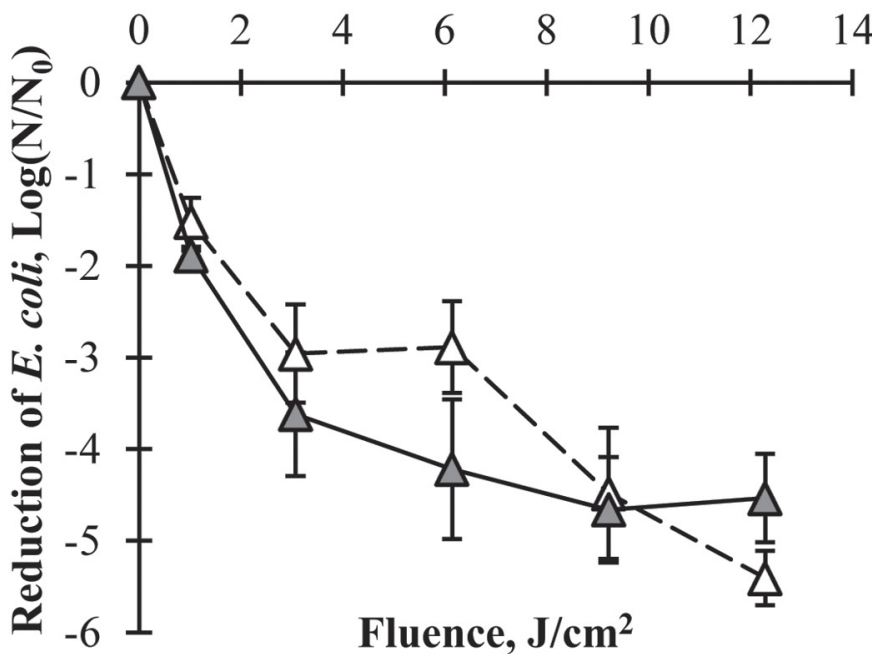

Figure 3. Pulsed-light inactivation of Escherichia coli ATCC 25922 on (a, b) Cheddar cheese and (c, d) process cheese, highlighting different combinations of initial inoculum levels ( $5 \mathrm{log}$ or $7 \mathrm{log} \mathrm{cfu} / \mathrm{slice}$ ) and presence (filled symbols) or absence (open symbols) of UV-transparent low-density polyethylene packaging. Survivor ratios were quantified as Log $\left(\mathrm{N} / \mathrm{N}_{0}\right)$, where $\mathrm{N}$ represents the number of recovered survivors (log cfu) and $\mathrm{N}_{0}$ represents the measured initial inoculum (log $\mathrm{cfu}$ ). The data points represent mean values of triplicates and the error bars indicate \pm 1 standard deviation.

$<0.05)$. Listeria innocua was the least sensitive to PL treatment, with a maximum inactivation level of 3.37 $\pm 0.2 \log$ (Figure 1), followed by P. fluorescens, with a maximum inactivation of $3.74 \pm 0.8 \log$ (Figure 2). In both cases, maximum inactivation levels were achieved on process cheese at an initial inoculum of $7 \log \mathrm{cfu} /$ slice, for treatments performed through packaging
(Figure 1d, Figure 2d). Lasabagaster and Martinez de Marañon (2012) observed the same order of bacterial sensitivity to PL using fish product isolates inoculated onto agar. Escherichia coli was the most sensitive to PL, with a maximum reduction of $5.41 \pm 0.1 \mathrm{log}$, achieved on process cheese at an initial inoculum of $7 \mathrm{log} \mathrm{cfu} /$ slice, without packaging (Figure 3d). 
Table 1. Significant predictors of Escherichia coli ATCC 35922, Listeria innocua, and Pseudomonas fluorescens inactivation by pulsed light (PL)

\begin{tabular}{llr}
\hline & Significant predictor & \\
of inactivation level & $P$-value \\
\hline E. coli ATCC 25922 & Cheese type $\times$ PL dose & $<0.0001$ \\
& Cheese type $\times$ packaging & $<0.0001$ \\
& Inoculum level $\times$ PL dose & 0.0369 \\
& Inoculum level $\times$ packaging & $<0.0001$ \\
L. innocua & Inoculum level $\times$ cheese type & 0.0058 \\
P. fluorescens & PL dose & $<0.0001$ \\
\hline
\end{tabular}

The different responses to PL of the 3 bacterial species can be explained by their respective protective mechanisms against UV light: L. innocua, P. fluorescens, and E. coli have inherent physiological properties that offer varying levels of protection to their nucleoid. For gram-positive bacteria such as L. innocua, the cell wall's thick peptidoglycan layer shields the DNA located in the cell's nucleus from PL lethal light discharges. The relatively low inactivation levels obtained in this study are consistent with previous reports: a reduction of $1.37 \mathrm{log} \mathrm{cfu} /$ sample on Vienna sausages was obtained by Uesugi and Moraru (2009), $0.9 \pm 0.09 \log$ reduction of $L$. monocytogenes on beef carpaccio was achieved by Hierro et al. (2012), and $1.41 \pm 0.15 \log$ reduction of L. monocytogenes on American cheese under similar conditions was reported by Can et al. (2014).

Pseudomonas fluorescens is a gram-negative bacteria that does not benefit from the protection of a thick peptidoglycan layer, but it has the ability to synthesize pyoverdin, a fluorescent blue pigment that absorbs light in the UV range (Brown and Luke, 2010), thus reducing the effective PL dose. In addition to this potential defense mechanism, P. fluorescens has the ability to form biofilms, which are complex matrices of extracellular polymeric substances that can offer physical protection to the enclosed bacterial population. Garvey et al. (2014) have shown that PL was only able to inactivate Pseudomonas aeruginosa in biofilms on surfaceinoculated agar at very high doses, much higher than the FDA-allowed doses. Out of all the effects tested in the general linear model, PL inactivation of $L$. innocua and P. fluorescens was only affected by PL dose. For $E$. coli, multiple interaction effects were found to be significant (Table 1).

Escherichia coli is a gram-negative microorganism that has been shown consistently to be more sensitive to PL compared with other pathogenic bacteria in various food matrices such as apple juice, orange juice (Pataro et al., 2011), and milk (Palgan et al., 2011). This greater sensitivity explains why the response of $E$. coli to PL was also the most influenced by the different treatment conditions. A significant cheese type $\times \mathrm{PL}$ dose interaction was primarily due to the fact that, at high doses, greater inactivation was achieved on process cheese, with a plateau at around 4-log reduction (Figure 3c, 3d), than on Cheddar cheese, for which a plateau was reached at around 3-log reduction (Figure $3 \mathrm{a}, 3 \mathrm{~b})$. This difference was slightly more pronounced at high inoculum levels (Figure 3b, Figure 3d), as highlighted by the significant inoculum level $\times$ cheese type interaction. Another interesting observation was the dependence between the type of cheese and the presence of LDPE packaging (Table 1), which will be discussed below.

\section{Effect of LDPE Packaging on PL Inactivation}

The potential of PL to be used on packaging materials and on packaged foods has been investigated before. Ringus and Moraru (2013) reported that PL treatment of L. innocua through transparent LDPE packaging was as effective as when applied directly on the inoculated side of the packaging material. Fernández et al. (2009) reported that PL consistently achieved the same degree of inactivation of $L$. monocytogenes on inoculated agar either unwrapped or wrapped in polyethylene, polyamide/polyethylene/vinyl acetate co-polymer, or polyamide/polyethylene copolymer.

The results of this study indicate that the presence of clear, UV transparent LDPE packaging did not interfere with PL treatment, regardless of the organism being treated. Moreover, the presence of an LDPE packaging layer seemed to increase the PL effectiveness against $E$. coli on Cheddar cheese (Figure 3b). The reasons for this increase in effectiveness are not clear at this point and need further investigation. Pulsed-light inactivation of L. monocytogenes on polypropylene-packaged American cheese also did not show significant differences from unpackaged samples (Can et al., 2014). This demonstrates the potential for PL treatment on cheese prepackaged in clear, UV-transparent packaging material.

\section{Effect of Cheese Surface Characteristics on PL Inactivation}

The effect of cheese surface characteristics was found to be insignificant $(P>0.05)$ for $L$. innocua and $P$. fluorescens (Table 1). However, for E. coli, high PL doses led to greater inactivation levels on process cheese than on Cheddar cheese (Figure 3), suggesting a higher surface exposure to PL on process cheese.

Although the surface of the 2 types of cheese used in this study appeared somewhat different to the naked eye, surface roughness analysis indicated that they were not statistically different in terms of average rough- 
Table 2. Surface characteristics of cheese samples

\begin{tabular}{lccccc}
\hline & \multicolumn{4}{c}{ Surface roughness parameter, ${ }^{1} \mu \mathrm{m}$} & Water contact \\
\cline { 2 - 5 } Substrate & $\mathrm{S}_{\mathrm{a}}$ & $\mathrm{S}_{\mathrm{z}}$ & $\mathrm{S}_{\mathrm{sk}}$ & $\mathrm{S}_{\mathrm{ku}}$ & ${ }^{\circ}$ \\
\hline Cheddar & $3.8 \pm 0.5$ & $30.2 \pm 4.2$ & $0.64 \pm 0.2$ & $3.3 \pm 0.5$ & $58.6 \pm 2.3^{\mathrm{a}}$ \\
Process cheese & $4.5 \pm 1.8$ & $31.3 \pm 6.8$ & $0.35 \pm 0.2$ & $2.9 \pm 0.9$ & $45.6 \pm 3.4^{\mathrm{b}}$ \\
\hline
\end{tabular}

${ }^{\mathrm{a}, \mathrm{b}}$ Within a column, different letters indicate significant differences $(P<0.05)$.

${ }^{1}$ Measured surface roughness parameters included the average surface roughness $\left(S_{a}\right)$, the difference between the highest and lowest peaks $\left(\mathrm{S}_{\mathrm{z}}\right)$, surface skewness $\left(\mathrm{S}_{\mathrm{sk}}\right)$, and surface kurtosis $\left(\mathrm{S}_{\mathrm{ku}}\right)$.

ness, highest to lowest peak height difference, surface skewness, or surface kurtosis (Table 2). However, topographic maps revealed that the surface of Cheddar cheese had a greater number of cavities per unit area than did that of process cheese (Figure 4). This particular detail was not captured by surface roughness analysis and could explain the higher inactivation achieved on process cheese at high doses, as the larger number of surface nonuniformities on Cheddar cheese offered more potential for microbial shading, especially at high inoculum concentrations.

Another metric used to compare substrate surface characteristics was the water contact angle, as a measure of surface hydrophobicity. The surface of Cheddar cheese had higher water contact angles and thus was more hydrophobic than process cheese (Table 2). These differences may be explained by the respective fat contents of the 2 cheeses: $32 \%$ for Cheddar versus $21 \%$ for process cheese. The difference in surface hydrophobicity indicates that the liquid inoculum tended to bead up on the surface of Cheddar cheese, which resulted in more cell clustering and light shading effects. This was less pronounced on process cheese, on which the liquid inoculum was able to spread more and thus avoid the shading effects described above.

\section{CONCLUSIONS}

Pulsed-light treatment was able to consistently achieve $>2$-log reductions of bacterial contaminants on $2.5 \times 5 \mathrm{~cm}$ cheese surfaces under a variety of conditions and at lower doses than the FDA-approved limit. The lack of interference from LDPE packaging highlights the opportunity to use PL as a terminal treatment on cheese prepackaged in clear, UV-transparent materials. Pulsed-light treatment could become a very attractive solution to mitigate surface contamination of cheese in manufacturing, distribution, and retail environments.

\section{ACKNOWLEDGMENTS}

This project was supported by the Dairy Research Institute (Rosemont, IL; project no. DRI-65385). This work made use of the Cornell Center for Materials Re-
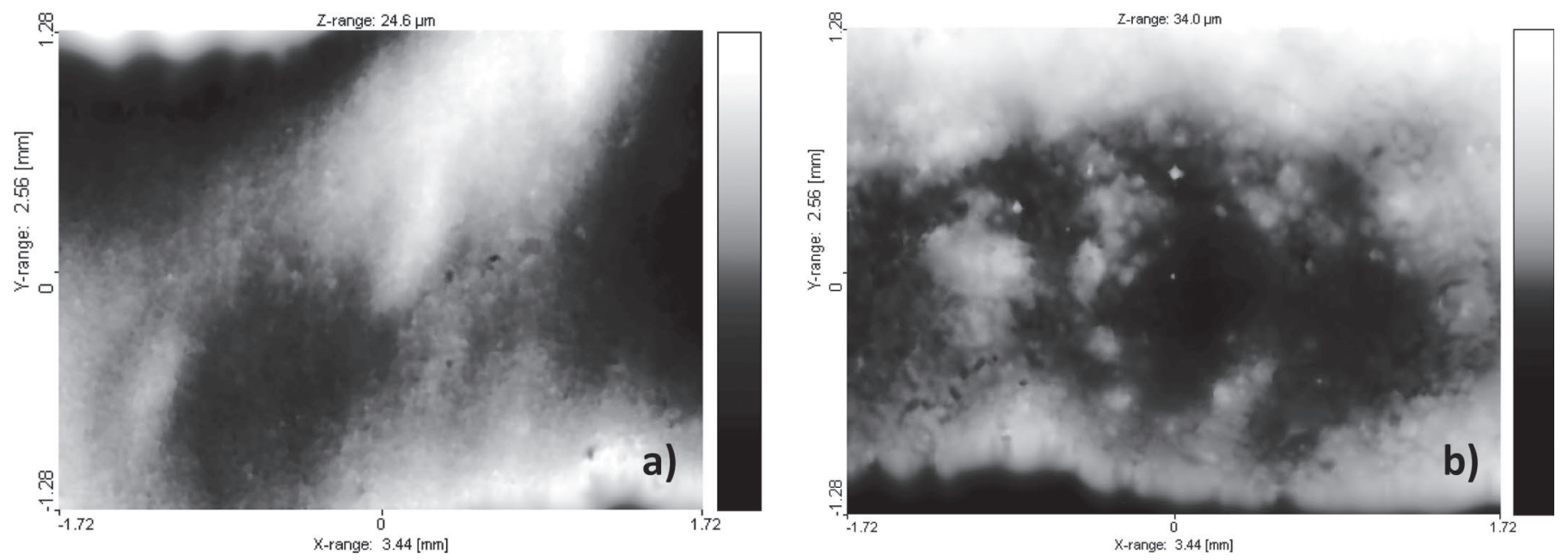

Figure 4. Topographic maps spanning $3.44 \mathrm{~mm}$ in the x-range and $2.56 \mathrm{~mm}$ in the y-range generated with an interferometric profiler of (a) process cheese sample, and (b) Cheddar cheese sample. The z-range indicates the highest peak in the spanned area. The white areas represent peaks and the dark areas represent valleys. 
search (CCMR) Shared Facilities (Cornell University, Ithaca, NY), supported through the National Science Foundation MRSEC program (DMR-1120296).

\section{REFERENCES}

Brown, A. G., and R. K. J. Luke. 2010. Siderophore production and utilization by milk spoilage Pseudomonas species. J. Dairy Sci. 93:1355-1363.

Can, F. O., A. Demirci, V. M. Puri, and H. Gourama. 2014. Decontamination of hard cheeses by pulsed UV light. J. Food Prot. 77:1723-1731

CDC (Centers for Disease Control and Prevention). 2012. Multistate outbreak of listeriosis linked to imported Frescolina Marte brand ricotta salata cheese (final update). Accessed Apr. 5, 2014. http:// www.cdc.gov/listeria/outbreaks/cheese-09-12/index.html?s_ cid $=$ fb1807.

Downes, F. P., K. Ito, and American Public Health Association. 2001. Compendium of Methods for the Microbiological Examination of Foods. 4th ed. American Public Health Association, Washington, DC.

Dunn, J., T. Ott, and W. Clark. 1995. Pulsed-light treatment of food and packaging. Food Technol. 49:95-98.

Elmnasser, N., S. Guillou, F. Leroi, N. Orange, A. Bakhrouf, and M. Federighi. 2007. Pulsed-light system as a novel food decontamination technology: A review. Can. J. Microbiol. 53:813-821.

Fernández, M., S. Manzano, L. de la Hoz, J. A. Ordóñez, and E. Hierro. 2009. Pulsed light inactivation of Listeria monocytogenes through different plastic films . Foodborne Pathog. Dis. 6:1265-1267.

Garvey, M., D. Rabbitt, A. Stocca, and N. Rowan. 2014. Pulsed ultraviolet light inactivation of Pseudomonas aeruginosa and Staphylococcus aureus biofilms. Water Environ J. 29:36-42. http://dx.doi. org/10.1111/wej.12088

Gomez-Lopez, V. M., P. Ragaert, J. Debevere, and F. Devlieghere. 2007. Pulsed light for food decontamination: A review. Trends Food Sci. Technol. 18:464-473.

Gould, L. H., E. Mungai, and C. Barton Behravesh. 2014. Outbreaks attributed to cheese: Differences between outbreaks caused by unpasteurized and pasteurized dairy products, United States, 19982011. Foodborne Pathog. Dis. 11:545-551.

Hierro, E., M. Ganan, E. Barroso, and M. Fernández. 2012. Pulsed light treatment for the inactivation of selected pathogens and the shelf-life extension of beef and tuna carpaccio. Int. J. Food Microbiol. 158:42-48.

Jackson, K. A., M. Biggerstaff, M. Tobin-D'Angelo, D. Sweat, R. Klos, J. Nosari, O. Garrison, E. Boothe, L. Saathoff-Huber, L. Hainstock, and R. P. Fagan. 2011. Multistate outbreak of Listeria monocytogenes associated with Mexican-style cheese made from pasteurized milk among pregnant, Hispanic women. J. Food Prot. 74:949-953.

Koch, J., R. Dworak, R. Prager, B. Becker, S. Brockmann, A. Wicke, H. Wichmann-Schauer, H. Hof, D. Werber, and K. Stark. 2010 Large listeriosis outbreak linked to cheese made from pasteurized milk, Germany, 2006-2007. Foodborne Pathog. Dis. 7:1581-1584.

Kramer, B., and P. Muranyi. 2014. Effect of pulsed light on structural and physiological properties of Listeria innocua and Escherichia coli. J. Appl. Microbiol. 116:596-611. http://dx.doi.org/10.1111/ jam.12394.

Lasagabaster, A., and I. Martinez de Marañon. 2012. Sensitivity to pulsed light technology of several spoilage and pathogenic bacteria isolated from fish products. J. Food Prot. 75:2039-2044.

Martin, N. H., S. C. Murphy, R. D. Ralyea, M. Wiedmann, and K. J. Boor. 2011. When cheese gets the blues: Pseudomonas fluorescens as the causative agent of cheese spoilage. J. Dairy Sci 94:3176-3183.

Miller, B. M., A. Sauer, and C. I. Moraru. 2012. Inactivation of Escherichia coli in milk and concentrated milk using pulsed-light treatment. J. Dairy Sci. 95:5597-5603.

Nogarol, C., P. L. Acutis, D. M. Bianchi, C. Maurella, S. Peletto, S. Gallina, and L. Decastelli. 2013. Molecular characterization of Pseudomonas fluorescens isolates involved in the Italian "blue mozzarella" event. J. Food Prot. 76:500-504.

Palgan, I., I. M. Caminiti, A. Muñoz, F. Noci, P. Whyte, D. J. Morgan, and J. G. Lyng. 2011. Effectiveness of high intensity light pulses (HILP) treatments for the control of Escherichia coli and Listeria innocua in apple juice, orange juice and milk. Food Microbiol. 28:14-20.

Pataro, G., A. Muñoz, I. Palgan, F. Noci, G. Ferrari, and J. G. Lyng. 2011. Bacterial inactivation in fruit juices using a continuous flow pulsed light (PL) system. Food Res. Int. 44:1642-1648.

Pereira, R. N., and A. A. Vicente. 2010. Environmental impact of novel thermal and non-thermal technologies in food processing. Food Res. Int. 43:1936-1943.

Ramos-Villarroel, A. Y., N. Aron-Maftei, O. Martín-Belloso, and R. Soliva-Fortuny. 2012. The role of pulsed light spectral distribution in the inactivation of Escherichia coli and Listeria innocua on fresh-cut mushrooms. Food Contr. 24:206-213.

Ringus, D. L., and C. I. Moraru. 2013. Pulsed light inactivation of Listeria innocua on food packaging materials of different surface roughness and reflectivity. J. Food Eng. 114:331-337.

Sauer, A., and C. I. Moraru. 2009. Inactivation of Escherichia coli ATCC 25922 and Escherichia coli $\mathrm{O} 157: \mathrm{H} 7$ in apple juice and apple cider, using pulsed light treatment. J. Food Prot. 72:937-944.

Ternström, A., A. M. Lindberg, and G. Molin. 1993. Classification of the spoilage flora of raw and pasteurized bovine milk, with special reference to Pseudomonas and Bacillus. J. Appl. Bacteriol. $75: 25-34$.

Uesugi, A. R., and C. I. Moraru. 2009. Reduction of Listeria on readyto-eat sausages after exposure to a combination of pulsed light and nisin. J. Food Prot. 72:347-353.

US FDA (Food and Drug Administration). 1996. Kinetics of microbial inactivation for alternative food processing technologies-pulsed light technology; final rule. 21CFR part 179.41. Accessed Mar. 3, 2013. http://www.ecfr.gov/cgi-bin/textidx?SID=57af6cfd4fc81035 640c1c1c78b95f19\&node=pt21.3.179\&rgn=div5\#se21.3.179_141.

Wang, T., S. J. MacGregor, J. G. Anderson, and G. A. Woolsey. 2005 Pulsed ultra-violet inactivation spectrum of Escherichia coli. Water Res. 39:2921-2925.

Woodling, S. E., and C. I. Moraru. 2005. Influence of surface topography on the effectiveness of pulsed light treatment for the inactivation of Listeria innocua on stainless steel surfaces. J. Food Sci. $70: 345-351$ 